\title{
Avaliação qualitativa das preparações de cardápios de unidades de alimentação e nutrição hospitalares em Sergipe
}

Qualitative evaluation of the menus preparations of Hospital Food Services in Sergipe

\author{
T. S. Lima'; E. R. S. A. Wartha'; A. C. Souza ${ }^{2}$; I. M. M. Carvalho ${ }^{3 *}$ \\ ${ }^{1}$ Departamento de Nutrição/Centro de Ciências Biológicas e da Saúde,Universidade Federal de Sergipe, 49100-000, \\ São Cristóvão-Sergipe, Brasil \\ ${ }^{2}$ Maternidade Nossa Senhora de Lourdes/Serviço de Nutrição e Dietética, 49080-470, Aracaju-Sergipe, Brasil \\ ${ }^{3}$ Departamento de Nutrição/Laboratório de Técnica Dietética/Centro de Ciências Biológicas e da \\ Saúde,Universidade Federal de Sergipe, 49100-000, São Cristóvão-Sergipe, Brasil \\ *i.montezano.c@gmail.com \\ (Recebido em 03 de fevereiro de 2019; aceito em 07 de junho de 2019)
}

\begin{abstract}
$\mathrm{O}$ presente trabalho objetivou avaliar a qualidade nutricional dos cardápios ofertados a funcionários e acompanhantes de pacientes em Unidades de Alimentação e Nutrição Hospitalares (UANH), localizadas no estado de Sergipe. Foram analisados os cardápios de sete UANH localizadas em Sergipe, ofertados a funcionários e acompanhantes de pacientes, nos almoços do mês de novembro de 2017. Para a análise, utilizou-se o método da Avaliação Qualitativa das Preparações do Cardápio - AQPC. De acordo com o método APQC, a maior parte dos cardápios apresentaram ofertas regulares em relação à presença de fruta $(42,9 \%)$, folhosos $(85,7 \%)$, monotonia de cores $(57,1 \%)$, alimentos ricos em enxofre $(85,7 \%)$ e doces $(42,9 \%)$. Já os critérios fritura, fritura associada a doces e conservas, apresentaram percentuais de ocorrência variando, predominantemente, entre bom e ótimo. Apesar dos resultados positivos, ofertas inadequadas ou próximas à inadequação foram observadas em algumas unidades, principalmente em relação à monotonia de cores, presença de alimentos ricos em enxofre, baixa oferta de frutas, ocorrência de conservas e doces, devendo estes, serem objetos de atenção quanto ao planejamento dos cardápios, em função dos possíveis efeitos deletérios ao organismo. Assim, cada Unidade deve buscar ajustar os diferentes aspectos que compõem os cardápios, sendo indispensável a atenção a todas as questões que envolvem uma alimentação saudável.

Palavras-chave: serviços de alimentação, planejamento de cardápios, análise qualitativa.
\end{abstract}

This paper aimed to evaluate the nutritional quality of the menus offered to hospital staff and companions of patients, in a Hospital Food Service (HFS), located in the state of Sergipe / Brazil. The menus of seven HFS located in Sergipe, offered to staff and companions of patients, at the lunches of November 2017 were analyzed. For the analysis, the method of Qualitative Evaluation of Menu Components - QEMC was used. According to the QEMC method, most of the menus presented regular offers in relation to fruit presence $(42.9 \%)$, leafy $(85.7 \%)$, color monotony $(57.1 \%)$, foods rich in sulfur $(85.7 \%)$ and sweet $(42.9 \%)$. The criteria frying, frying associated with sweets and preserves, presented percentage of occurrence varying, predominantly, between good and optimum. In spite of the positive results, inadequate or close to inadequate supplies were observed in some units, mainly in relation to color monotony, presence of foods rich in sulfur, low supply of fruits, preserves and sweets, and these should be objects of attention to the planning of the menus, due to the possible deleterious effects that they cause to the organism. Therefore, each Unit should seek to adjust the different aspects that make up the menus, being indispensable to attend to all questions that involve a healthy diet.

Keywords: food services, menu planning, qualitative analysis.

\section{INTRODUÇÃO}

Os hábitos alimentares das populações sofreram intensas modificações, em função das alterações no estilo de vida, devido ao crescente processo de urbanização e industrialização [1]. Estas mudanças levaram à prática cada vez mais constante da alimentação fora do lar, e assim, ao desenvolvimento do setor de alimentação coletiva, com consequente aumento do número de Unidades de Alimentação e Nutrição (UAN) [2]. 
As UAN são estabelecimentos de produção e distribuição de refeições tanto para indivíduos saudáveis, quanto para enfermos [3], e podem estar localizadas em diversas instituições, tendo como objetivo o fornecimento de uma alimentação adequada do ponto de vista nutricional e higiênico-sanitário, que satisfaça às demandas dos usuários [4, 5]. Entre as UAN, a UAN Hospitalar (UANH) é apontada como um dos setores mais importantes da instituição onde está inserida, uma vez que é peça chave no processo dietoterápico, além de fornecer refeições para acompanhantes dos pacientes internados e funcionários [6].

A oferta de refeições nutricionalmente adequadas em ambientes de trabalho é de extrema importância, levando em consideração que os trabalhadores passam boa parte do seu dia em seus locais de trabalho, onde muitos realizam duas das principais refeições do dia. Além disso, o local de trabalho configura um espaço de compartilhamento de informações e convívio social, representando um local estratégico para a promoção da saúde e hábitos alimentares saudáveis [7, 8].

Entretanto, ao contrário do que se espera, dados da literatura vêm demonstrando a presença de inadequações nos cardápios produzidos por UANH, como monotonia em relação à oferta de alimentos, preparações, características sensoriais e nutricionais em cardápios destinados à alimentação dos trabalhadores $[9,10]$. Tais inadequações podem refletir negativamente na aceitação das refeições e repercutir no estado nutricional dos clientes, sejam eles pacientes ou funcionários. Vale ressaltar que, no caso dos funcionários, a baixa aceitação dos cardápios pode refletir negativamente no seu estado nutricional, uma vez que a maioria realiza ao menos duas refeições diárias no local de trabalho, devido às jornadas laborais características do ambiente hospitalar [8].

Nesse contexto, uma alimentação desequilibrada pode comprometer a produtividade de trabalhadores, prejudicar o sistema de defesa contra patologias (o que assume particular importância pelo fato de atuarem em ambiente hospitalar), bem como aumentar os riscos de acidentes de trabalho [11, 12]. Além disso, este desequilíbrio pode representar um risco para o desenvolvimento de doenças crônicas não transmissíveis (DCNT), como a obesidade, hipertensão e diabetes [13].

Levando em consideração os fatores apresentados e a busca pelo planejamento adequado de cardápios, existem recursos que auxiliam na elaboração e avaliação da composição qualitativa desses, como o método de Avaliação Qualitativa de Preparações do Cardápio (AQPC), que permite a avaliação de cardápios através dos itens que os compõem [14]. Deste modo, o AQPC visa, além de contribuir na construção de cardápios equilibrados do ponto de vista nutricional e sensorial, auxiliar na identificação de erros na elaboração dos mesmos, permitindo assim a correção destes antes mesmo da sua execução [15].

Diante do exposto, o objetivo do presente estudo foi avaliar a qualidade nutricional dos cardápios ofertados a funcionários e acompanhantes, em UANH localizadas no estado de Sergipe.

\section{MATERIAL E MÉTODOS}

Trata-se de um estudo transversal, no qual foram avaliados cardápios planejados em Unidades de Alimentação e Nutrição localizadas em hospitais no estado de Sergipe. Foi avaliado o cardápio mensal de cada UANH, planejado para o almoço de 30 dias, durante o mês de novembro de 2017, ofertado a funcionários e acompanhantes de pacientes dos hospitais. A análise do cardápio referente a um mês completo ocorreu para controlar o erro aleatório da medida dietética, que poderia decorrer da presença de um dia não característico do padrão de planejamento do cardápio.

A seleção da amostra foi estabelecida por conveniência, onde apenas as instituições que atenderam a todas às solicitações participaram da pesquisa. Para esta seleção, inicialmente foi realizada uma pesquisa on-line do número de hospitais em funcionamento localizados nas regionais de saúde do estado de Sergipe (Aracaju, Estância, Itabaiana, Lagarto, Nossa Senhora da Glória, Nossa Senhora do Socorro e Propriá) [16].

Posteriormente, foi realizado o primeiro contato via e-mail e/ou por telefone com os responsáveis pelas Unidades, a fim de apresentar a pesquisa e verificar a disponibilidade para participação. Aos responsáveis que aceitaram participar, foi solicitado o cardápio do mês de 
novembro de 2017, ofertado a funcionários e acompanhantes de pacientes do hospital em que a UANH estava localizada, juntamente com o preenchimento de um questionário de caracterização composto por seis perguntas (tipo de hospital, profissional responsável técnico da unidade, tipo de serviço, número de refeições, tipo de cardápio e modalidade de distribuição das refeições).

A participação foi efetivada a partir da assinatura do Termo de Consentimento Livre e Esclarecido (TCLE). De 18 instituições hospitalares localizadas em Sergipe, sete concordaram em participar da pesquisa.

Para análise dos cardápios foram utilizadas as informações contidas nos mesmos, sendo que as dúvidas referentes a questões como modo de preparo e ingredientes utilizados, foram sanadas pelo questionamento direto ao responsável técnico pelo cardápio.

A análise dos cardápios foi realizada a partir do método AQPC, adaptado de Veiros e Proença [15], sendo analisados os seguintes itens: oferta de folhosos, frutas in natura como sobremesa, monotonia de cores, preparações ricas em enxofre (exceto feijão), doces como sobremesa, frituras, doce e fritura em um mesmo dia e conservas. Não foi possível realizar a avaliação dos cardápios quanto à oferta de carne gordurosa, devido à impossibilidade de acesso às fichas técnicas de preparação das refeições, uma vez que a maioria das unidades não fazia uso de tal instrumento, configurando-se, portanto, uma limitação da pesquisa.

Foi considerada a ocorrência de monotonia de cores quando duas ou mais preparações apresentavam as mesmas cores predominantes. Foram consideradas preparações ricas em enxofre, quando havia duas ou mais ocorrências com um dos seguintes ingredientes: abacate, acelga, aipo, alho, amendoim, batata-doce, brócolis, castanha, cebola, couve-de-bruxelas, couve-flor, ervilha, gengibre, goiaba, jaca, lentilha, maçã, melancia, melão, milho, mostarda, nabo, nozes, ovo, rabanete, repolho e uva. Os cardápios também foram avaliados quanto à oferta diária de frutas, doces, frituras, folhosos, doces associados a frituras e conservas [15, 17].

Analisou-se a presença de fritura isoladamente e quando associada à oferta de doces servidos como sobremesas, como pudins, gelatinas, mousses, sorvetes e afins. Cada critério foi avaliado por ocorrência diária, posteriormente semanal, e por último mensal, sendo o resultado obtido em porcentagem, considerando o percentual de ocorrência em um total de 30 dias.

Para classificação de cada item avaliado, foram aplicados os critérios utilizados por Prado et al. (2013) [18]. A oferta de frutas e folhosos foi avaliada e classificada de acordo com os seguintes critérios: ótimo $\geq 90 \%$; bom $75 \%$ a $89 \%$; regular de $50 \%$ a $74 \%$; ruim de $25 \%$ a $49 \%$; péssimo $<25 \%$. A presença de alimentos ricos em enxofre, monotonia das cores, fritura, doce, doce e fritura no mesmo dia e conservas foram definidos como itens de aspecto negativo, e, a partir do percentual de ocorrência foram classificadas em ótimo $\leq 10 \%$; bom de $11 \%$ a $25 \%$; regular de $26 \%$ a $50 \%$; ruim de $51 \%$ a $75 \%$; e péssimo $>75 \%$.

Os dados foram tabulados pelo programa Microsoft Exce $^{\circledR}{ }^{\circledR}$ 2010, e para a análise estatística, utilizaram-se representações descritivas e tabelas de frequência.

\section{RESULTADOS E DISCUSSÃO}

Os dados referentes às características gerais das sete UANH participantes encontram-se na Tabela 1.

Todas as Unidades participantes localizavam-se em instituições hospitalares públicas e sob modalidade de gestão terceirizada. Os padrões dos cardápios variaram entre simples $(28,6 \%)$ e médio $(71,4 \%)$. Foram considerados cardápios de padrão simples aqueles que ofertavam apenas uma opção de prato principal, e de padrão médio, os que ofertavam pelo menos duas opções de pratos proteicos como prato principal. A distribuição das refeições era realizada de maneira porcionada e em balcão térmico em todas as UANH, e o número diário de almoços ofertados pelas UANHs variou entre 30 a 700 refeições. Além disso, todas as unidades tinham como responsável técnico um profissional nutricionista presente no local. 
Tabela 1. Características das Unidades de Alimentação e Nutrição Hospitalar (UANH).

\begin{tabular}{ccccccc}
\hline UANH & $\begin{array}{c}\text { Tipo de } \\
\text { hospital }\end{array}$ & $\begin{array}{c}\text { Tipo de } \\
\text { serviço }\end{array}$ & $\begin{array}{c}\text { Responsável } \\
\text { técnico }\end{array}$ & $\begin{array}{c}\mathbf{N}^{\mathbf{0}} \text { Diário de } \\
\text { Almoços }\end{array}$ & $\begin{array}{c}\text { Padrão de } \\
\text { Cardápio }\end{array}$ & $\begin{array}{c}\text { Sistema de } \\
\text { Distribuição }\end{array}$ \\
\hline A & Público & Terceirizado & Nutricionista & 43 & Simples & $\begin{array}{c}\text { Cafeteria } \\
\text { fixa }\end{array}$ \\
\hline B & Público & Terceirizado & Nutricionista & 30 & Médio & $\begin{array}{c}\text { Cafeteria } \\
\text { fixa }\end{array}$ \\
\hline C & Público & Terceirizado & Nutricionista & 700 & Médio & $\begin{array}{c}\text { Cafeteria } \\
\text { fixa }\end{array}$ \\
\hline D & Público & Terceirizado & Nutricionista & 180 & Médio & $\begin{array}{c}\text { Cafeteria } \\
\text { fixa }\end{array}$ \\
\hline E & Público & Terceirizado & Nutricionista & 250 & Médio & $\begin{array}{c}\text { Cafeteria } \\
\text { fixa }\end{array}$ \\
\hline F & Público & Terceirizado & Nutricionista & 132 & Simples & $\begin{array}{c}\text { Cafeteria } \\
\text { fixa }\end{array}$ \\
\hline G & Público & Terceirizado & Nutricionista & 150 & Médio & $\begin{array}{c}\text { Cafeteria } \\
\text { fixa }\end{array}$ \\
\hline
\end{tabular}

O nutricionista, na realização das suas funções, deve garantir a oferta de refeições adequadas do ponto de vista nutricional, levando em consideração todos os aspectos que envolvem a alimentação, contribuindo desta forma para a recuperação e promoção da saúde [11]. Desse modo, o planejamento adequado de cardápios em UANs se mostra um instrumento importante, visto que, por meio deste, é possível contribuir para a oferta equilibrada de nutrientes, bem como auxiliar na formação de hábitos alimentares saudáveis da clientela [19, 20].

Os resultados da análise qualitativa dos cardápios pelo método AQPC encontram-se na Tabela 2.

Tabela 2. Análise da ocorrência diária dos critérios analisados pelo método Avaliação Qualitativa de Preparações do Cardápio (AQPC) durante o mês de novembro de 2017 em Unidades de Alimentação e Nutrição Hospitalares (UANH) no estado de Sergipe.

\begin{tabular}{ccccccccc}
\hline & \multicolumn{7}{c}{ Critério avaliado } \\
\cline { 2 - 9 } UANH & $\begin{array}{c}\text { Frutas } \\
(\boldsymbol{\%})\end{array}$ & $\begin{array}{c}\text { Folhosos } \\
(\boldsymbol{\%})\end{array}$ & $\begin{array}{c}\text { Cores } \\
\text { iguais } \\
(\boldsymbol{\%})\end{array}$ & $\begin{array}{c}\text { Ricos em } \\
\text { enxofre } \\
(\boldsymbol{\%})\end{array}$ & $\begin{array}{c}\text { Doce } \\
(\boldsymbol{\%})\end{array}$ & $\begin{array}{c}\text { Fritura } \\
(\boldsymbol{\%})\end{array}$ & $\begin{array}{c}\text { Fritura } \\
+ \text { Doce } \\
(\boldsymbol{\%})\end{array}$ & $\begin{array}{c}\text { Conserva } \\
(\boldsymbol{\%})\end{array}$ \\
\hline $\mathbf{A}$ & 0 & 70,0 & 56,7 & 43,3 & 0 & 20,0 & 0 & 6,7 \\
$\mathbf{B}$ & 73,3 & 50,0 & 46,7 & 50,0 & 26,7 & 20,0 & 13,3 & 26,7 \\
$\mathbf{C}$ & 66,7 & 73,3 & 43,3 & 46,7 & 33,3 & 20,0 & 6,6 & 13,3 \\
$\mathbf{D}$ & 56,7 & 63,3 & 23,3 & 46,7 & 43,3 & 13,3 & 10,0 & 0 \\
$\mathbf{E}$ & 33,3 & 50 & 23,3 & 63,3 & 66,7 & 16,7 & 16,7 & 66,7 \\
$\mathbf{F}$ & 0 & 83,3 & 36,7 & 46,7 & 0 & 10,0 & 0 & 0 \\
$\mathbf{G}$ & 100 & 63,3 & 50,0 & 50,0 & 0 & 20,0 & 0 & 10,0 \\
\hline
\end{tabular}

Observa-se que a maior parte das UANHs apresentou oferta aquém do desejado para frutas, sendo que apenas em $14,3 \%$ foi constatada uma ótima oferta (100\% de ocorrência), enquanto $42,9 \%$ apresentaram uma oferta regular, $14,3 \%$, ruim, e $28,6 \%$, nenhuma oferta (péssima). Entre as principais frutas ofertadas estavam a banana $(20 \%)$, a laranja $(16,3 \%)$ e o mamão $(9,4 \%)$. Apenas em 14,3\% das UANHs a oferta de folhosos foi classificada como "boa", com presença em $83,3 \%$ dos dias. Nas demais $(85,7 \%)$, a oferta foi classificada como regular, com resultados entre $50 \%$ e $73,3 \%$, sendo acelga (25\%), repolho $(15,3 \%)$ e alface $(10,2 \%)$ os principais folhosos encontrados. 
O consumo diário de frutas e vegetais é indispensável ao bom funcionamento do organismo, sendo estes alimentos as principais fontes de vitaminas, fibras e minerais [21, 22]. Um hábito alimentar que inclui estes grupos de alimentos está relacionado à redução do risco para o desenvolvimento de DCNT, enquanto seu consumo insuficiente relaciona-se a deficiências nutricionais, distúrbios de peso e enfermidades correlatas [23, 24].

Resultados diferentes aos encontrados no presente estudo foram demonstrados em investigação realizada por Resende e Quintão (2016) [14], em UAN localizada em uma empresa privada em Leopoldina - MG, onde se observou oferta de frutas e folhosos em 78,86\% e 86,53\% dos dias no cardápio ofertado aos trabalhadores, respectivamente. Já em estudo realizado por Said et al. (2014) [25] em UAN localizada em restaurante universitário em Belém - PA, foi observada elevada oferta de frutas $(100 \%)$, mas baixa oferta de vegetais folhosos $(8,69 \%)$. No contexto de UANHs, Brito et al. (2013) [10] encontraram resultados semelhantes aos do presente trabalho para a oferta de hortaliças folhosas e frutas, isto é, abaixo do desejável para estes grupos de alimentos.

De acordo com a análise da monotonia de cores, $14,3 \%$ das unidades apresentaram cores iguais em 56,7\% dos dias, recebendo a classificação ruim, e as demais $(85,7 \%)$ foram classificadas entre regular e bom, com aparecimento deste item em $23,3 \%$ a $50 \%$ dos dias.

A presença de similaridade de cores de alimentos ofertados pode afetar negativamente a escolha dos alimentos e a satisfação dos clientes com a refeição, desestimulando a vontade de consumir os alimentos [16]. Para ser classificado como adequado, um cardápio deve apresentar variedade e harmonia. A variedade se caracteriza pela diversidade de ingredientes, consistências, cores e sabores, tornando a refeição mais atrativa à comensalidade. Já a harmonia é observada pela combinação de cores, texturas e sabores, o que requer sentido artístico e estético $[14,26]$. Tal diversificação das cores auxilia na garantia de ingestão de diferentes nutrientes, além de despertar o interesse do consumidor, considerando que o primeiro contato com o alimento é visual, proporcionando, desta forma, uma refeição mais prazerosa e promovendo a saúde do cliente $[3,23]$.

Estudo realizado por Benvindo et al. (2017) [27] em restaurantes universitários do Brasil, constatou a ocorrência de monotonia de cores superior a $46 \%$ em todos os cardápios analisados. Resultado semelhante foi encontrado por São José [2], em UAN localizada em Vitória - ES, sendo observado um percentual de ocorrência de 50,98\%. Já Ferreira et al. (2015) [3] verificaram monotonia de cores em 53\% dos dias analisados, em estudo realizado em UAN localizada em empresa privada do município de Santo Antônio de Jesus - BA. No âmbito das UANH, Petry et al. (2014) [11] encontraram monotonia de cores (presença de $\geq 3$ preparações com cores iguais) em $42 \%$ de um total de 19 dias analisados.

A presença de alimentos ricos em enxofre foi observada em 14,3\% das Unidades estudadas, com ocorrência em $63,3 \%$ dos dias analisados, sendo classificada como ruim. Os demais cardápios $(85,7 \%)$ receberam a classificação regular, com a presença de alimentos sulfurados variando entre $43,3 \%$ e $50 \%$, sendo os alimentos com maior frequência acelga (25\%), repolho $(15,3 \%)$ e cebola $(9,7 \%)$, ofertados principalmente em saladas.

Os alimentos ricos em enxofre podem ocasionar desconforto abdominal devido ao elevado teor de compostos sulfurados, e por este motivo, a presença de dois ou mais alimentos ricos neste nutriente deve ser evitada em uma mesma refeição [14, 19]. Estudos realizados em UANs localizadas em empresas privadas, como o de Ramos et al. (2011) [22] em Belo Horizonte - MG, constataram a presença de alimentos ricos em enxofre em $76,2 \%$ dos dias analisados. Prado et al. (2012) [18] em estudo realizado em UAN de médio porte localizada em uma empresa privada em Cuiabá - MT, observaram um percentual de ocorrência em $40 \%$ dos dias.

Com relação à presença de frituras, todas as unidades apresentaram um percentual de ocorrência entre $10 \%$ e $20 \%$, recebendo classificações entre bom $(85,7 \%)$ e ótimo $(14,3 \%)$, apresentando, desta forma, um aspecto positivo na avaliação.

A fritura é uma técnica de cocção rápida, normalmente escolhida para minimizar o tempo de preparo de alguns alimentos, bem como proporcionar aspectos sensoriais agradáveis às preparações. Entretanto, sabe-se que os alimentos ricos em gorduras, fornecem lipídios ao 
organismo, sendo estes, fontes potenciais de energia que contribuem para o aumento acentuado da densidade calórica da refeição $[10,11]$. Além disso, o consumo excessivo de lipídios saturados é considerado um fator de risco para o desenvolvimento de doenças cardiovasculares [2].

Resultados superiores aos encontrados no presente estudo foram observados por Brito et al. (2013) [10] e Petry et al. (2014) [11], em UANHs de Vitoria da Conquista - BA e Florianópolis - SC, onde a presença de fritura foi observada em $41 \%$ e $53 \%$ dos dias avaliados, respectivamente.

A oferta de doces como sobremesa foi observada em 14,3\% das unidades, com percentual de ocorrência de $66,7 \%$, recebendo a classificação ruim. Para o mesmo parâmetro, $42,9 \%$ das UANHs receberam a classificação regular, onde a oferta de doces ocorreu em 26,7\% a 43,3\% dos dias. Nas demais Unidades (42,9\%) não houve oferta de doces, recebendo a classificação "ótimo", sendo que destas, $28,6 \%$ não faziam oferta de nenhum tipo de sobremesa (frutas ou doces). Valor superior ao do presente estudo foi encontrado em UANH localizada em Vitória da Conquista BA, onde foi observada presença de doces no cardápio em 64,5\% dos dias [10].

Dados do Guia Alimentar para População Brasileira [28] preconizam que o açúcar apresenta um valor calórico superior, de cinco a dez vezes, ao da maioria das frutas. Além disso, o consumo de frutas em substituição aos doces elaborados apresenta diversos benefícios à saúde em função da sua composição [16]. Sabe-se que uma elevada oferta de doces está associada ao aumento do valor energético de refeições, bem como ao desenvolvimento de sobrepeso, obesidade, diabetes, hipertrigliceridemia, hipercolesterolemia e aumento da fração LDL-colesterol [10, 29].

Ao se avaliar a ocorrência da oferta de fritura e doce em um mesmo dia, observou-se que todas as UANHs apresentaram resultados positivos, sendo que $71,4 \%$ receberam a classificação "ótimo", com um percentual $\leq 10 \%$. Cabe ressaltar que $28,6 \%$ destas não faziam oferta de nenhum tipo de sobremesa (frutas ou doces). As demais $(28,6 \%)$ foram classificadas como "bom", com uma oferta entre $13,3 \%$ e $16,7 \%$ dos dias.

Alimentos ricos em carboidratos e lipídios, a depender da porção, podem desequilibrar nutricionalmente um cardápio, sendo normalmente limitados a uma variedade pequena de nutrientes. Desta forma, recomenda-se que seus consumos sejam realizados de forma moderada [28]. O consumo rotineiro de tais alimentos aumenta o risco de desenvolvimento de aterosclerose e outras patologias coronarianas [10]. Além disso, por apresentarem alto valor energético, quando consumidos excessivamente, podem levar ao ganho de peso e acúmulo de gordura corporal, podendo contribuir com o desenvolvimento de DCNT [14].

Resende e Quintão (2016) [14], ao analisar o cardápio de uma UAN institucional, encontraram presença de fritura e doce associados em 6,73\% dos dias. Por outro lado, Brito et al. (2013) [10] verificaram, em UANH, um percentual de ocorrência em 51,6\% dos dias analisados.

Em relação à presença de conservas nos cardápios, $57,1 \%$ das UANHs avaliadas receberam a classificação "ótimo", com percentual de ocorrência $\leq 10 \% ; 28,6 \%$ foram classificadas entre "bom" e "regular", com oferta variando de 13,3\% a 26,7\%; e 14,3\% receberam classificação "ruim", com a presença de conservas em 66,7\% dos dias analisados. Ramos et al. (2013) [22] verificaram, em uma UAN localizada em empresa privada, a presença de conservas em 33,3\% dos dias analisados, enquanto, Benvindo et al. (2017) [27] observaram em restaurantes universitários do Brasil, valores inferiores a $16 \%$ em todos os cardápios estudados.

As conservas são alimentos que trazem em sua composição elevadas quantidades de sódio, ingrediente utilizado com objetivo de auxiliar na preservação e agregação de sabor do produto [16]. Entretanto, sabe-se que o consumo excessivo de sódio está relacionado ao desenvolvimento de patologias como hipertensão arterial e câncer gástrico [2, 30]. Desta forma, faz-se necessário a utilização limitada deste tipo de alimento [28].

\section{CONCLUSÃO}

Diante dos resultados apresentados, pode-se observar que a maior parte dos cardápios analisados apresentou percentuais de ocorrência que variaram entre "bom" e "ótimo", com oferta reduzida de fritura, fritura associada a doces, e conservas, sendo este um aspecto positivo da avaliação. Já a oferta de frutas, folhosos, monotonia de cores, alimentos ricos em enxofre e doces, apresentaram classificação predominantemente "regular". 
Entretanto, mesmo recebendo tais classificações, muitos foram os resultados que se aproximaram de um percentual de ocorrência "ruim", principalmente em relação à presença de monotonia de cores e alimentos ricos em enxofre, sendo estes, sinais de alerta para o cuidado no planejamento dos cardápios. Além disso, algumas UANHs apresentaram resultados ruins e péssimos para alguns dos critérios analisados, como a oferta de frutas, conservas e doces, havendo a necessidade de reformulações, visando à melhoria dos aspectos nutricionais, levando em consideração os efeitos prejudiciais que o consumo desequilibrado de tais alimentos podem trazer.

\section{REFERÊNCIAS BIBLIOGRÁFICAS}

1. Leal D. Crescimento da alimentação fora do domicílio. Seg Alim Nutr. 2010 Jan;17(1):123-132.

2. São José JFB. Avaliação qualitativa de cardápios em uma unidade de alimentação e nutrição localizada em Vitória-ES. Demetra: Alim Nutr Saúde. 2014 Dez;9(4):975-984.

3. Ferreira MSB, Vieira RB, Fonseca KZ. Aspectos quantitativos e qualitativos das preparações de uma Unidade de Alimentação e Nutrição em Santo Antônio de Jesus, Bahia. Nutrivisa: Rev Nutr Vig Saúde. 2015 Mar-Jun;2(1):22-27.

4. Machado M, Martins MAA, Andrade GQP, Kraemer FB. A qualidade nutricional e sensorial de preparações proteicas de unidades de alimentação e nutrição escolares: uma avaliação pelo método AQNS. Demetra: Alim Nutr Saúde. 2012;7(3):145-156.

5. Estevam E, Guimarães M. Caracterização do perfil nutricional e dos aspectos ergonômicos relacionados ao trabalho de colaboradores de uma unidade de alimentação e nutrição. Rev Cient Faminas. 2013 MaiAgo;9(2):55-68.

6. Ribeiro FP, Boni BR. Análise do processo de distribuição das dietas livres servidas aos pacientes de uma unidade de alimentação e nutrição hospitalar. Hig Alim. 2016 Mai-Jun;30(256):152-156.

7. Mattos, PF. Avaliação da Adequação do Almoço de uma Unidade de Alimentação e Nutrição (UAN) ao Programa de Alimentação do Trabalhador (PAT). Cadernos UniFOA. 2008 Ago;4(7):54-59.

8. Silva SB, Spinelli MGN. Consumo de frutas em unidade de alimentação e nutrição no município de São Paulo: um estudo de caso. Rev Univap. 2015 Dez;21(38):5-14.

9. Melo MTSM, Carvalho NPB, Campos CMF, Alencar MSS, Nunes IFOC. Aspectos dietéticos e nutricionais dos cardápios produzidos por uma unidade de alimentação hospitalar. Hig Alim. 2016 NovDez;30(262):21-25.

10. Brito LF, Bezerra VM. Avaliação qualitativa das preparações do cardápio de uma unidade de alimentação e nutrição hospitalar de vitória da conquista, Bahia. Braz J Food Nutr. 2013 Abr-Jun; 24(2):153-158.

11. Petry NS, Veloso RGSS, Pulz IS, Cebola M, Veiros MB. Avaliação e proposta de novos cardápios para a ceia de uma unidade de alimentação e nutrição hospitalar pública de Florianópolis (SC). Demetra: Alim Nutr Saúde. 2014 Dez; 9(4):903-924.

12. Lagemann MD, Fassina P. Avaliação da composição nutricional de refeições oferecidas em uma unidade de alimentação e nutrição. Rev Cad Pedag. 2015;12(1):36-45.

13. Simon MISS, Garcia CA, Lino ND, Forte GC, Fontoura ID, Oliveira ABA. Avaliação nutricional dos profissionais do serviço de nutrição e dietética de um hospital terciário de Porto Alegre. Cad Saude Colet. 2014 Jan-Mar;22(1):69-74.

14. Resende FR, Quintão DF. Avaliação qualitativa das preparações do cardápio de uma unidade de alimentação e nutrição institucional de Leopoldina-MG. Demetra: Alim Nutr Saúde. 2016;11(1):91-98.

15. Veiros MB, Proença RPC. Avaliação Qualitativa das Preparações do Cardápio em uma Unidade de Alimentação e Nutrição - Método AQPC. Rev Nutr Pauta. 2003 Set-Out;11(62):36-42.

16. IBGE. Disponível em: URL: https://cidades.ibge.gov.br/brasil/se/panorama.

17. Proença RPC, Hering B, Sousa AA, Veiros MB. Qualidade nutricional e sensorial na produção de refeições. Florianópolis: UFSC; 2005. p. 91-92.

18. Prado BG, Nicoletti AL, Farias CS. Avaliação qualitativa das preparações de cardápio em uma unidade de alimentação e nutrição de Cuiabá- MT. UNOPAR Cient Ciênc Biol Saúde. 2013 Mai; 15(3):219-23.

19. Rodrigues AGM, Proença RP, Calvo MC, Fiates GM. Overweight/obesity is associated with food choices related to rice and beans, colors of salads, and portion size among consumers at a restaurant serving buffet-by-weight in Brazil. Appetite. 2012, 59(2):305-11.

20. Santos JS. Análise qualitativa e quantitativa do cardápio semanal de um serviço de alimentação e nutrição de um hospital particular, localizado na cidade de Salvador, Bahia. Rev Assoc Bras NutrRASBRAN. 2016 Jul-Dez;7(2):100-105. 
21. Silva SB, Spinelli MGN. Consumo de frutas em unidade de alimentação e nutrição no município de São Paulo: um estudo de caso. Rev Univap. 2015 Dez;21(38):5-14.

22. Ramos AS, Souza FFR, Fernandes GCB, Xavier SKP. Avaliação de cardápio e pesquisa de satisfação. Braz J Food Nutr. 2013 Jan-Fev;24(1):29-35.

23. Melo PF, Tinoco LPC, Alves VR. Relação entre o estado nutricional e o consumo de frutas, verduras e legumes de pacientes atendidos em uma clínica escola de nutrição. Ensaios e Ciência: Ciências Biológicas, Agrárias e da Saúde 2011 Mar;15(4):117-130.

24. Muniz LC, Zanini RDV, Schneider BC, Tassitano RM, Feitosa WMDN, González-Chica DA. Prevalência e fatores associados ao consumo de frutas, legumes e verduras entre adolescentes de escolas públicas de Caruaru, PE. Cien Saude Colet. 2013 Fev;18: 393-404.

25. Said NM, Santos GBC, Santos MSP, Silva EB. Avaliação qualitativa do cardápio de um restaurante universitário de Belém do Pará. Anais do III Congresso de Educação em Saúde da Amazônia. Belém: Universidade Federal do Pará; 2014.

26. Ornellas LH. Técnica dietética: seleção e preparo dos alimentos. 8 ed. São Paulo: Atheneu; 2006. 309 p.

27. Benvindo JLS, Souza AMP, Bandoni DH. Qualidade nutricional de cardápios planejados para restaurantes universitários de universidades federais do Brasil. Demetra: Alim Nutr Saúde. 2017; 12(2):447-464.

28. Brasil. Ministério da Saúde. Secretaria de Atenção à Saúde. Departamento de Atenção Básica. Guia alimentar para a população brasileira. 2. ed. Brasília: Ministério da Saúde; 2014. 156 p.

29. Silva KF, Drumond N, Quintão DF. Avaliação do perfil nutricional e condições de trabalho de colaboradores de unidades de alimentação e nutrição de Eugenópolis (MG). Rev Cient Faminas. 2015 Mai-Ago;11(2): 33-48.

30. World Health Organization. Forum on reducing salt intake in populations. Reducing salt intake in populations: report of a WHO forum and technical meeting; 5-7 Oct. 2006, Paris, France. Geneva: WHO; 2006. 